\title{
A Belgian View on Lattice Rules
}

\author{
Ronald Cools ${ }^{1}$ and Dirk Nuyens ${ }^{2}$
}

1 Dept. of Computer Science, K.U. Leuven, Celestijnenlaan 200A, B-3001 Heverlee, Belgium

Ronald.Cools@cs.kuleuven.be

2 Dept. of Computer Science, K.U. Leuven, Celestijnenlaan 200A, B-3001 Heverlee, Belgium

Dirk.Nuyens@cs.kuleuven.be

\section{Introduction}

The problem we consider is the approximation of multivariate integrals over the $s$-dimensional unit cube

$$
I[f]:=\int_{0}^{1} \cdots \int_{0}^{1} f\left(x_{1}, \ldots, x_{s}\right) \mathrm{d} x_{1} \cdots \mathrm{d} x_{s}=\int_{[0,1)^{s}} f(\mathbf{x}) \mathrm{d} \mathbf{x} .
$$

We are interested in approximations of the form

$$
Q[f]:=\sum_{j=1}^{n} w_{j} f\left(\mathbf{y}^{(j)}\right)
$$

with weights $w_{j} \in \mathbb{R}$ and points $\mathbf{y}^{(j)} \in[0,1)^{s}$.

Many people call this a quadrature problem, although strictly speaking the word "quadrature" refers to the 1-dimensional case, i.e., measuring an area. By only using this key word in a search, one misses a whole world of relevant literature. The more appropriate word is "cubature". In written English, it appears already in the 17 th century to refer to measuring a volume. ${ }^{3}$ Because one speaks about an $s$-dimensional cube, it is natural to use the same word in connection with measuring $s$-dimensional volumes, i.e., integrals. So, if $s=1$ then $Q$ is called a quadrature formula and if $s \geq 2$ then $Q$ is called a cubature formula.

We are particularly interested in cubature formulas where the points $\mathbf{y}^{(j)}$ and weights $w_{j}$ are chosen independent of the integrand $f$. It is usually difficult and time consuming to construct such cubature formulas, but the result is usually hard coded in programs or tables.

\footnotetext{
${ }^{3}$ An equivalent exists in other languages, e.g., in German "Kubatur" and in Dutch "kubatuur".
} 
In the taxonomy of cubature formulas one can distinguish two major classes: polynomial based methods (e.g., methods exact for algebraic or trigonometric polynomials) and number theoretic methods (e.g., quasi-Monte Carlo methods and even Monte Carlo methods based on pseudo random number generators). As in zoology, some species are difficult to classify. Lattice rules are a family of cubature formulas that are studied as members of both classes, depending on the background of the researcher. They are in the focus of this text.

Definition 1. An s-dimensional lattice rule is a cubature formula which can be expressed in the form

$$
Q[f]=\frac{1}{d_{1} d_{2} \ldots d_{t}} \sum_{j_{1}=1}^{d_{1}} \sum_{j_{2}=1}^{d_{2}} \ldots \sum_{j_{t}=1}^{d_{t}} f\left(\left\{\frac{j_{1} \mathbf{z}_{1}}{d_{1}}+\frac{j_{2} \mathbf{z}_{2}}{d_{2}}+\ldots+\frac{j_{t} \mathbf{z}_{t}}{d_{t}}\right\}\right)
$$

where $t$ and $d_{i} \in \mathbb{N} \backslash\{0\}$ and $\mathbf{z}_{i} \in \mathbb{Z}^{s}$ for all $i$.

The notation $\{\cdot\}$ denotes to take the fractional part componentwise.

An alternative definition is given below. This already shows that lattice rules can be approached in different ways.

Definition 2. A multiple integration lattice $\Lambda$ is a subset of $\mathbb{R}^{s}$ which is discrete and closed under addition and subtraction and which contains $\mathbb{Z}^{s}$ as a subset. A lattice rule is a cubature formula where the $n$ points are the points of a multiple integration lattice $\Lambda$ that lie in $[0,1)^{s}$ and the weights are all equal to $1 / n$.

We must emphasize that a lattice rule has different representations of the form given in Definition 1. The minimal number of sums (i.e., the minimal number of generating vectors $\mathbf{z}_{i}$ ) required is called the rank of the lattice rule. Even if the number of generators is fixed, the rules can still be represented using different generating vectors. Many papers only consider lattice rules of rank 1. A rank-1 lattice rule is generated by one vector $\mathbf{z}$ and has the form

$$
Q[f]=\frac{1}{n} \sum_{j=1}^{n} f\left(\left\{\frac{j \mathbf{z}}{n}\right\}\right)
$$

The view on lattice rules presented in this text is strongly biased. It reflects how the first author got into contact with lattice rules, and how he started looking at them from the view on multivariate integration he had at that time. (For a different view on lattice rules, which also includes other kinds of quasiMonte Carlo point sets, we refer to [LL02].) In Section 2 an overview of quality and construction criteria for lattice rules is given, biased towards what is less known in the qMC-world, i.e., the target audience of this volume. In Section 3 we will briefly describe recent approaches for constructing lattice rules, making it clear that the choice of quality criterion determines the required construction effort. In Section 4 we will point to techniques to make lattice rules work in practice and in Section 5 we will illustrate that lattice rules are used from 2-dimensions to high dimensions. Final remarks are given in Section 6 . 


\section{Quality Criteria}

\subsection{Rules Exact for Polynomials}

There are many quality criteria to specify and classify cubature formulas in general, and lattice rules in particular. Trigonometric polynomials play an important role in the world of lattice rules. Algebraic polynomials play a role in connection with more "classical" cubature formulas. In this section we will point to some similarities.

Let $\mathbf{h}=\left(h_{1}, h_{2}, \ldots, h_{s}\right) \in \mathbb{Z}^{s}$ and $|\mathbf{h}|:=\sum_{j=1}^{s}\left|h_{j}\right|$. An algebraic polynomial is a finite sum of the form

$$
p(\mathbf{x})=\sum_{\mathbf{h} \in \mathbb{Z}^{s}} a_{\mathbf{h}} \mathbf{x}^{\mathbf{h}}=\sum_{\mathbf{h} \in \mathbb{Z}^{s}} a_{\mathbf{h}} \prod_{j=1}^{s} x_{j}^{h_{j}}, \quad \text { with } h_{j} \geq 0 .
$$

A trigonometric polynomial is a finite sum of the form

$$
t(\mathbf{x})=\sum_{\mathbf{h} \in \mathbb{Z}^{s}} a_{\mathbf{h}} e^{2 \pi i \mathbf{h} \cdot \mathbf{x}}=\sum_{\mathbf{h} \in \mathbb{Z}^{s}} a_{\mathbf{h}} \prod_{j=1}^{s} e^{2 \pi i x_{j} h_{j}} .
$$

The degree of a polynomial is defined as $\max _{a_{\mathbf{h}} \neq 0}|\mathbf{h}|$. The space of all algebraic polynomials in $s$ variables of degree at most $d$ is denoted by $\mathbb{P}_{d}^{s}$. The space of all trigonometric polynomials in $s$ variables of degree at most $d$ is denoted by $\mathbb{T}_{d}^{s}$. We will use the symbol $\mathbb{V}_{d}^{s}$ to refer to one of the vector spaces $\mathbb{P}_{d}^{s}$ or $\mathbb{T}_{d}^{s}$.

The dimensions of the vector spaces of polynomials are

$$
\operatorname{dim} \mathbb{P}_{d}^{s}=\left(\begin{array}{c}
s+d \\
d
\end{array}\right) \quad \text { and } \quad \operatorname{dim} \mathbb{T}_{d}^{s}=\sum_{j=0}^{s}\left(\begin{array}{l}
s \\
j
\end{array}\right)\left(\begin{array}{l}
d \\
j
\end{array}\right) 2^{j}
$$

The right hand sides are polynomials in $d$ of degree $s$.

A very old quality criterion for cubature formulas comes from demanding that the formula gives the exact value of the integral for polynomials.

Definition 3. A cubature formula $Q$ has algebraic (trigonometric) degree $d$ if it is exact for all polynomials of algebraic (trigonometric) degree at most $d$.

Once this criterion is put forward, it is natural to ask how many points are needed in a cubature formula to obtain a specified degree of precision. This is obviously related to the dimension of the space for which the formula reproduces the exact value of the integral.

Theorem 1. If a cubature formula is exact for all polynomials of $\mathbb{V}_{2 k}^{s}$, then the number of points $n \geq \operatorname{dim} \mathbb{V}_{k}^{s}$.

A proof of this result for algebraic degree is given in [Rad48] for $s=2$ and in [Str60] for general $s$. For trigonometric degree it is presented in [Mys87]. So, the required number of points increases exponentially with the dimension. Furthermore a "large" part of the weights in a cubature formula have to be positive. 
Theorem 2. If a cubature formula is exact for all polynomials of $\mathbb{V}_{d}^{s}$ and has only real points and weights, then it has at least $\operatorname{dim} \mathbb{V}_{k}^{s}$ positive weights, $k=\left\lfloor\frac{d}{2}\right\rfloor$.

This result is proven in [Mys81] for algebraic degree and [Coo97] for trigonometric degree. The combination of the two previous theorems implies that formulas attaining the lower bound of Theorem 1 have only positive weights. For trigonometric degree, we even know more [BC93].

Corollary 1. If a cubature formula of trigonometric degree $2 k$ has $n=\operatorname{dim} \mathbb{T}_{k}^{s}$ points, then all weights are equal.

One cannot expect that the lower bound of Theorem 1 can be attained for odd degrees $2 k+1$, since in that case it is equal to the bound for degree $2 k$. For algebraic degree, there exists an improved lower bound for odd degrees that takes into account information on the symmetry of the integration region. The first such result was derived for centrally symmetric regions such as a cube. For surveys of achievements in this particular area we refer to [Coo97, CMS01]. A similar result holds for the trigonometric degree case. Let $G_{k}$ be the span of trigonometric monomials of degree $\leq k$ with the same parity as $k$.

Theorem 3. The number of points $n$ of a cubature formula for the integral over $[0,1)^{s}$ which is exact for all trigonometric polynomials of degree at most $d=2 k+1$ satisfies

$$
n \geq 2 \operatorname{dim} G_{k}
$$

This result is mentioned in [Nos85] and a complete proof appears in [Mys87].

Structures do not only play a role in the derivation of lower bounds; their role in constructing cubature formulas is even more important. Imposing structure on the points and weights is used since the beginning of history to reduce the complexity of the construction problem for cubature formulas. The basic structure for lattice rules is "shift symmetry". In the trigonometric case this structure plays the same role as "central symmetry" in the algebraic case.

Definition 4. A cubature formula is called shift symmetric if it is invariant with respect to the group of transformations

$$
\left\{\mathrm{x} \mapsto \mathrm{x}, \mathrm{x} \mapsto\left\{\mathrm{x}+\left(\frac{1}{2}, \ldots, \frac{1}{2}\right)\right\}\right\}
$$

Hence, the multiple integration lattice $\Lambda$ of a shift symmetric cubature formula satisfies

$$
\left\{\mathrm{x}+\left(\frac{1}{2}, \ldots, \frac{1}{2}\right) \mid \mathrm{x} \in \Lambda\right\}=\Lambda \text {. }
$$

This structure was exploited to derive the following result [BC93].

Theorem 4. If a shift symmetric cubature formula of degree $2 k+1$ has $n=$ $2 \operatorname{dim} G_{k}$ points, then all weights are equal. 
In the algebraic case it is proven that formulas attaining the lower bound for odd degrees for centrally symmetric regions are also centrally symmetric. For the trigonometric case it was conjectured in [Coo97].

The results of Corollary 1 and Theorem 4 motivate us to restrict searches for cubature formulas of trigonometric degree to equal weight cubature formulas. Hence the general form (1) simplifies to

$$
Q[f]=\frac{1}{n} \sum_{j=1}^{n} f\left(\mathbf{y}^{(j)}\right) .
$$

Formulas for which the lower bounds in Theorems 1 and 3 are sharp, are only known for degrees 1, 2 and 3 in all dimensions, for all degrees in 2 dimensions, and for degree 5 in 3 dimensions. We refer to [Coo97, Lyn03] for a detailed survey. Almost all known formulas of trigonometric degree that attain these lower bounds are (shifted) lattice rules. The only exceptions are derived in [CS96].

Theorem 5. The following points

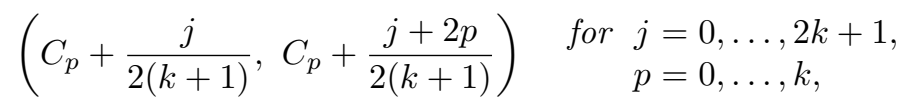

with $C_{0}=0$ and $C_{1}, \ldots, C_{k}$ arbitrary, are the points of a cubature formula for the integral over $[0,1)^{2}$ of trigonometric degree $2 k+1$.

We are not aware of successful efforts to construct cubature formulas of trigonometric degree that are not (shifted) lattice rules. In Section 3 we will mention recent construction methods for lattice rules with the trigonometric degree criterion, not necessarily attaining the known lower bounds.

Most of the results summarized above were obtained using reproducing kernels, see [Aro50]. A reproducing kernel $K$ is in general a function of two $s$-dimensional variables with the property that an evaluation of a function $f$ can be written as the inner product of $f$ with $K$. If we work in a finite dimensional space of polynomials, then a reproducing kernel can be written using orthogonal polynomials. The trigonometric case is easier to work with than the algebraic case because orthonormal polynomials are readily available. Indeed, the trigonometric monomials form an orthonormal sequence. A further simplifying aspect of the trigonometric case is that the reproducing kernel can be written as a function of one $s$-dimensional variable. For $s=2$ and $\mathbb{T}_{d}^{s}$ it has a simple form which was exploited in [CS96] to obtain formulas with the lowest possible number of points, including lattice rules and others (see Theorem 5).

\subsection{On Route to Other Quality Criteria}

So far, we focused on integrating polynomials. What do we know if we apply a cubature formula to a function that is not a polynomial? To answer this 
question, let us assume the integrand function $f$ can be expanded into an absolutely convergent multiple Fourier series:

$$
f(\mathbf{x})=\sum_{\mathbf{h} \in \mathbb{Z}^{s}} \hat{f}(\mathbf{h}) e^{2 \pi i \mathbf{h} \cdot \mathbf{x}} \quad \text { with } \quad \hat{f}(\mathbf{h}):=\int_{[0,1)^{s}} f(\mathbf{x}) e^{-2 \pi i \mathbf{h} \cdot \mathbf{x}} \mathrm{d} \mathbf{x} .
$$

Then the approximation error of an equal weight cubature formula (2) is given by

$$
\begin{aligned}
Q[f]-I[f] & =\frac{1}{n} \sum_{j=1}^{n}\left(\sum_{\mathbf{h} \in \mathbb{Z}^{s} \backslash\{\mathbf{0}\}} \hat{f}(\mathbf{h}) e^{2 \pi i \mathbf{h} \cdot \mathbf{y}^{(j)}}\right) \\
& =\sum_{\mathbf{h} \in \mathbb{Z}^{s} \backslash\{\mathbf{0}\}}\left(\hat{f}(\mathbf{h}) \frac{1}{n} \sum_{j=1}^{n} e^{2 \pi i \mathbf{h} \cdot \mathbf{y}^{(j)}}\right) .
\end{aligned}
$$

Observe that

$$
\frac{1}{n} \sum_{j=1}^{n} e^{2 \pi i \mathbf{h} \cdot \mathbf{y}^{(j)}}= \begin{cases}1 & \text { if } \mathbf{h} \cdot \mathbf{y}^{(j)} \in \mathbb{Z} \\ 0 & \text { if } \mathbf{h} \cdot \mathbf{y}^{(j)} \notin \mathbb{Z} .\end{cases}
$$

So, if our equal weight cubature formula is a lattice rule, many terms in the expression for the error vanish. This brings us to a very important tool to investigate the error of a lattice rule and a well known theorem by Sloan and Kachoyan [SK87].

Definition 5. The dual of the multiple integration lattice $\Lambda$ is

$$
\Lambda^{\perp}:=\left\{\mathbf{h} \in \mathbb{Z}^{s}: \mathbf{h} \cdot \mathbf{x} \in \mathbb{Z} \forall \mathbf{x} \in \Lambda\right\} .
$$

Theorem 6. Let $\Lambda$ be a multiple integration lattice. Then the corresponding lattice rule $Q$ has an error

$$
Q[f]-I[f]=\sum_{\mathbf{h} \in \Lambda^{\perp} \backslash\{\mathbf{0}\}} \hat{f}(\mathbf{h}) .
$$

Remember that our analysis in this section assumes that the integrand can be expanded in an absolutely convergent multiple Fourier series. So, lattice rules look interesting for periodic functions. Not surprisingly, the trigonometric degree can be defined in terms of the dual lattice.

Definition 6. The trigonometric degree of a lattice rule $Q$ is

$$
d(Q):=\min _{\mathbf{h} \in \Lambda^{\perp} \backslash\{\mathbf{0}\}}\left(\sum_{j=1}^{s}\left|h_{j}\right|\right)-1 .
$$


For many years this criterion was only used in Russia for construction. Some references are [Mys85, Mys90, Rez90, Nos85, Nos88, Tem91, Sem96, Sem97, Osi04, OP04].

Another popular criterion for lattice rules that can also be defined in terms of the dual lattice is the Zaremba index or figure of merit.

Definition 7. The Zaremba index or figure of merit is

$$
\rho(Q):=\min _{\mathbf{h} \in \Lambda^{\perp} \backslash\{\mathbf{0}\}}\left(\bar{h}_{1} \bar{h}_{2} \cdots \bar{h}_{s}\right) \quad \text { with } \quad \bar{h}:=\max (1,|h|) .
$$

The Zaremba index was used in a computer search for good lattice rules in three and four dimensions by Maisonneuve [Mai72], and also in, e.g., [BP85]. The now classical survey [Lyn89] already presented both the Zaremba index and the trigonometric degree (there it is called "overall degree") in the form of the above definitions.

We will now sketch the origin of the Zaremba index. For $c>0$ and fixed $\alpha>1$, let $E_{s}^{\alpha}(c)$ be the class of functions $f$ whose Fourier coefficients satisfy

$$
|\hat{f}(\mathbf{h})| \leq \frac{c}{\left(\bar{h}_{1} \bar{h}_{2} \cdots \bar{h}_{s}\right)^{\alpha}} .
$$

This is essentially a class of functions of a certain smoothness, given by $\alpha$. The worst possible function in class $E_{s}^{\alpha}(1)$ is

$$
f_{\alpha}(\mathbf{x}):=\sum_{\mathbf{h} \in \mathbb{Z}^{s}} \frac{1}{\left(\bar{h}_{1} \bar{h}_{2} \cdots \bar{h}_{s}\right)^{\alpha}} e^{2 \pi i \mathbf{h} \cdot \mathbf{x}} .
$$

Now define $P_{\alpha}(Q)$ as the error of the lattice rule $Q$ for the function $f_{\alpha}$ :

$$
P_{\alpha}(Q):=\sum_{\mathbf{h} \in \Lambda^{\perp} \backslash\{\mathbf{0}\}} \frac{1}{\left(\bar{h}_{1} \bar{h}_{2} \cdots \bar{h}_{s}\right)^{\alpha}} .
$$

When $\alpha$ is an even integer $P_{\alpha}(Q)$ is easy to compute because in that case $f_{\alpha}$ can be written as a product of Bernoulli polynomials. It was introduced by Korobov [Kor59] who showed the existence of lattice rules for which $P_{\alpha}(Q)$ is $O\left(n^{-\alpha+\epsilon}\right), \epsilon>0$, or $O\left(n^{-\alpha}(\log (n))^{\alpha s}\right)$ in [Kor60].

It follows easily that the larger $\rho(Q)$ is, the smaller we expect $P_{\alpha}(Q)$ to be; the $\mathbf{h}$ which achieve the minimum in the definition of $\rho(Q)$ make up the largest value in the sum for $P_{\alpha}(Q)$. A lower bound on $P_{\alpha}(Q)$ can easily be derived from the definitions as

$$
\frac{2}{\rho(Q)^{\alpha}} \leq P_{\alpha}(Q)
$$

but the real use of $\rho(Q)$ is in deriving upper bounds, see, e.g., [Nie78, Nie92] for an overview. 
Another related criterion is given by

$$
R(Q):=\sum_{\substack{\mathbf{h} \in \Lambda^{\perp} \backslash\{\mathbf{0}\} \\-\frac{n}{2}<h_{j} \leq \frac{n}{2}}} \frac{1}{\left(\bar{h}_{1} \bar{h}_{2} \cdots \bar{h}_{s}\right)} .
$$

Here, Fourier coefficients which are already at a certain distance from the origin are not considered anymore. This has the benefit that no smoothness parameter $\alpha$ has to be chosen. In other words: $R(Q)$ is a modified version of $P_{\alpha}$, chosen in such a way that $\alpha$ can be set to one. A similar lower bound as for $P_{\alpha}(Q)$ is given by

$$
\frac{1}{\rho(Q)} \leq R(Q)
$$

Recent searches based on $R(Q)$ were done by Joe [Joe04] and Sinescu and Joe [JS07]. These searches are in fact searches for the "star discrepancy" by using a nice relationship in terms of $R(Q)$. (Loosely speaking, a point set has low discrepancy if the points are fairly well uniformly distributed in relation to the number of points used, see, e.g., [Nie78, Nie92].) For large $n$ it can be inferred that large values of $\rho(Q)$ will give small values of both $R(Q)$ and $P_{\alpha}(Q)$.

In a Korobov space with smoothness $\alpha$ the value of $P_{\alpha}(Q)$ is the square of the worst-case error. The worst-case error of a cubature rule $Q$ in a space $\mathcal{F}$ is given by

$$
e(Q, \mathcal{F})=\sup _{\substack{f \in \mathcal{F} \\\|f\|_{\mathcal{F}} \leq 1}}|I(f)-Q(f)|
$$

Such a Korobov space is a reproducing kernel Hilbert space (see [Aro50]). As was already mentioned, a reproducing kernel Hilbert space is a function space for which the evaluation of a function can be written as the inner product with the reproducing kernel $K$. This reproducing kernel is in general a function of two variables, but when the function space is periodic the kernel can in fact be written as a function in one variable. Such a kernel is then called shift-invariant. For a shift-invariant kernel $K$ and a rank-1 lattice the squared worst-case error is given by

$$
e^{2}(Q, K)=-\int_{[0,1)^{s}} K(\mathbf{x}, \mathbf{0}) \mathrm{d} \mathbf{x}+\frac{1}{n} \sum_{j=1}^{n} K\left(\left\{\frac{j \mathbf{z}}{n}\right\}, \mathbf{0}\right),
$$

see, e.g., [Hic98a]. So if one knows the reproducing kernel, one can obtain an explicit formula for the worst-case error in the space under consideration.

A very important and recent ingredient in these reproducing kernel Hilbert spaces are weights which are used to denote the importance of certain sets of variables. (Note that these weights are different from the ones in the cubature formula (1).) The most simple and useful form of the kernel is the kernel for a 
shift-invariant and tensor-product weighted reproducing kernel Hilbert space. In this case the kernel can be written as

$$
K(\mathbf{x}, \mathbf{y})=\prod_{k=1}^{s}\left(1+\gamma_{k} \omega\left(\left\{x_{k}-y_{k}\right\}\right)\right) .
$$

The weights $\gamma_{k} \geq 0$ are used to denote the importance of the different dimensions. For a rank-1 rule the typical form for the squared worst-case error in such a weighted space is then

$$
e_{s}^{2}(\mathbf{z})=-1+\frac{1}{n} \sum_{j=1}^{n} \prod_{k=1}^{s}\left(1+\gamma_{k} \omega\left(\left\{\frac{j z_{k}}{n}\right\}\right)\right),
$$

where we assumed that $\int_{0}^{1} \omega(x) \mathrm{d} x=0$.

The use of reproducing kernel Hilbert spaces has created a very elegant theory in which all kinds of discrepancies can be defined in terms of the worstcase error in a certain space (see, e.g., [Hic98a, Hic98b]). Moreover, it enables the study of the error in non-periodic spaces, see, e.g., [SKJ02a, SKJ02b].

\section{Recent Constructions}

In Section 2 we presented lower bounds for the number of points that is required in a cubature formula to attain a specified trigonometric degree. The theorems are not constructive and - as mentioned in Section 2-formulas that attain the known bounds are only known for small $s$ or $d$. The construction of lattice rules is done by searches. The parameters in such a search are the number of points, the number of generating vectors and the components of these vectors. Obviously the complete search space is huge. Furthermore, the cost to verify that a lattice rule has trigonometric degree $d$ is proportional to $d^{s}$. Consequently only "moderate" dimensions are feasible for this criterion for this reason only.

Practical constructions of lattice rules start with restricting the search space. A popular restriction is to consider only rank-1 lattice rules with one generating vector, hence only $s$ components have to be determined. Actually, most authors only consider so-called rank- 1 simple rules, where the first component of the generating vector is equal to 1 . Then only $s-1$ components have to be determined.

The search space can be even further reduced by considering only generator vectors of the form

$$
\mathbf{z}(\ell)=\left(1, \ell, \ell^{2} \bmod n, \ldots, \ell^{s-1} \bmod n\right), \quad 1<\ell<n .
$$

This is the form of so-called Korobov rules [Kor60].

In the remainder of this section we will sketch two recent successful types of searches. They use a different quality criterion and have their own way to restrict the search space. 


\subsection{Rules of Exact Trigonometric Degree}

Many searches for lattice rules use the generator matrix of the dual lattice. We will first properly introduce this concept. Recent searches impose some structure on this matrix.

Any $s$-dimensional lattice $\Lambda$ can be specified in terms of $s$ linearly independent vectors $\left\{\mathbf{a}_{1}, \mathbf{a}_{2}, \ldots, \mathbf{a}_{s}\right\}$. These vectors are known as generators of $\Lambda$. (In addition to the $t$ vectors in Definition 1 , one can always take $s-t$ unit vectors.) Associated with the generators is an $s \times s$ generator matrix $A$ whose rows are $\mathbf{a}_{1}, \mathbf{a}_{2}, \ldots, \mathbf{a}_{s}$. All lattice points $\mathbf{x}$ are of the form $\mathbf{x}=\sum_{i=1}^{s} \lambda_{i} \mathbf{a}_{i}=\boldsymbol{\lambda} A$ for some $\boldsymbol{\lambda} \in \mathbb{Z}^{s}$.

The dual lattice $\Lambda^{\perp}$ has generator matrix $B=\left(A^{-1}\right)^{T}$. Some authors use this as the definition of a dual lattice, instead of Definition 5 . They are equivalent. It can be shown that the number of points $n=|\operatorname{det} A|^{-1}=|\operatorname{det} B|$.

Recent searches in low dimensions are based on the following argument by [CL01]: "It is reasonable to believe that the lattice $\Lambda$ of an optimal lattice rule will have $\Lambda^{\perp}$ with many elements on the boundary of $S\left(O_{s}, d+1\right)$ ". Here $S\left(O_{s}, d+1\right)$ denotes a magnification of the unit octahedron $O_{s}$ by a factor $d+1$. Their searches only consider lattice rules whose dual has $s$ generators lying on the boundary of $S\left(O_{s}, d+1\right)$. The corresponding lattice rules are called $K$-optimal rules.

The cost for searching this space mainly depends on the number of generator matrices that must be considered; this is $O\left(d^{s^{2}-s}\right)$. Most of these can be eliminated quickly but for a minute proportion one has to verify their trigonometric degree, at a cost over $d^{s-1}$. This leads to a complexity bounded above by $d^{s^{2}-1}$. This is a pessimistic bound, but it indicates the fundamental problem of this approach. In [CL01] it was used for 3 and 4 dimensions. In [LS06] the results of a 'Seti@home'-type of search is described for 5 dimensions, limited to $d \leq 11$.

One can impose structure on the generator matrix of the dual lattice to reduce the number of free parameters in the search. In [LS04, CG03] the search was restricted to (skew-) circulant generator matrices. This reduces the cost to $O\left(d^{2 s-2}\right)$ and was very successful in $4-6$ dimensions. This approach also lead to closed expressions for lattice rules of arbitrary degrees. A more detailed summary of this approach is presented in [CN06]

We will conclude this part with a digression: linking the search for lattice rules to the field known as "geometry of numbers". To compare the number of points of different lattice rules of the same degree we require a proper scaling. The packing factor provides this.

Definition 8. The packing factor is

$$
\hat{\rho}(n):=\frac{(d+1)^{s}}{s ! n} .
$$

The packing factor is a measure of the efficiency of a rule and provides a convenient way for making pictures because $0 \leq \hat{\rho}(n) \leq 1$. 
Actually, $\hat{\rho}(n)$ is bounded above by what people working in the area of "geometry of numbers" call the density of the densest lattice packing of the crosspolytope (octahedron) $\theta\left(O_{s}\right)$ [GL87]. This provides a better lower bound for lattice rules for trigonometric degree than those of Theorems 1 and 3 :

$$
n \geq \frac{(d+1)^{s}}{s ! \theta\left(O_{s}\right)} .
$$

The problem is however that $\theta\left(O_{s}\right)$ is only known for $s=1,2$ and 3: $\theta\left(O_{1}\right)=$ $\theta\left(O_{2}\right)=1, \theta\left(O_{3}\right)=\frac{18}{19}$. This last result is due to Minkowski [Min67] and was already used in [Fro77] to construct lattice rules.

Lattice rules provide constructive lower bounds for $\theta\left(O_{s}\right)$. From a lattice rule with $n$ points having degree $d$ follows

$$
\theta\left(O_{s}\right) \geq \frac{(d+1)^{s}}{s ! n} .
$$

The currently best known bounds for $\theta\left(O_{s}\right), s=4,5$ and 6 all follow from known lattice rules [OP04, Coo06].

\subsection{Rules Minimizing a Worst-Case Error}

The introduction of weights in the function space, e.g., as in (4), makes it practically impossible to hard code the cubature rules in tables since there are an infinite number of weighted function spaces to choose from. The weights give the flexibility to tune the function space, but at a price. Luckily, for shift-invariant spaces we are able to construct lattice rules just in time by a fast algorithm.

If one wants to search lattice rules which minimize $P_{\alpha}(Q), R(Q)$ or any other weighted worst-case error for a given function space, then again the search space has to be limited in one way or another. In this section the focus will be on rank-1 rules. A traditional approach was to consider Korobov rules (5), but more recently, the component-by-component construction [SR02] has opened many more possibilities. Since the publication of [SR02] a lot of results concerning component-by-component construction were obtained, both on the existence and on the construction side, see, e.g., [SKJ02a, SKJ02b, DK04a, Kuo03, CKN06].

Instead of trying to find an optimal generating vector of a predefined form, the components of the generating vector are now searched, and fixed, component by component. In this way the complexity of the search is reduced from $O\left(n^{s} \kappa(n, s)\right)$ to $O(\operatorname{sn} \kappa(n, s))$ where $\kappa(n, s)$ is the cost of calculating the worst-case error by formula (4). By inspection we find that $\kappa(n, s)=O(s n)$ and thus the total cost is $O\left(s^{2} n^{2}\right)$. However, simply considering the product as a cumulative product, since the previous components of $\mathbf{z}$ are fixed, reduces the construction cost to $O\left(s n^{2}\right)$ at the expense of $O(n)$ memory. This allows for moderately larger values of $s$ and $n$ than for the exactness criteria, but really large values are still infeasible unless more advanced arguments are used. 
In [DK04a, DK04b] Dick and Kuo conceive a modified method to find lattice rules with "millions of points", for which $n$ needs to be a product of few primes. But even without modifying the search it turns out to be possible to construct lattice rules with millions of points and in thousands of dimensions. This was first shown by the authors for $n$ prime in [NC06a] and later extended for any composite $n$ [NC06b]. This fast algorithm allows for the construction of lattice rules on a just in time basis.

The fast algorithm works by exploiting some structural properties in the worst-case error formula. Starting from (4) it can be observed that the $\omega$ function is evaluated on a multiplicative algebraic structure modulo $n$

$$
\omega\left(\left\{\frac{j z}{n}\right\}\right)=\omega\left(\frac{j \cdot z \bmod n}{n}\right) .
$$

By rewriting (4) as a matrix-vector product it can be shown that a matrixvector multiplication with a matrix with the above structure can be done in $O(n \log n)$ using fast Fourier transforms, see [NC06b]. Therefore, construction takes only $O(s n \log (n))$ using $O(n)$ memory.

\section{Toward Using Lattice Rules}

Many texts start with saying that lattice rules are for integrating periodic functions. The different quality criteria we mentioned before make that clear. The traditional line of thought is that one first has to transform the region to the unit cube and then periodize the function. Periodization is further discussed in Section 4.1.

However, it is nowadays known that lattice rules can successfully be applied to non-periodic functions as well, see, e.g., [SKJ02a, SKJ02b]. In Section 4.2 we describe a recent trend in which lattice rules are even used as a sequence. Both these new insights reduce the historical differences between low discrepancy sequences and lattice rules.

\subsection{Periodizing Transformations}

A non-periodic function on the unit cube can be transformed by a periodizing transform $\phi$ :

$$
\int_{[0,1)^{s}} f\left(x_{1}, \ldots, x_{s}\right) \mathrm{d} \mathbf{x}=\int_{[0,1)^{s}} f\left(\phi\left(x_{1}\right), \ldots, \phi\left(x_{s}\right)\right) \phi^{\prime}\left(x_{1}\right) \cdots \phi^{\prime}\left(x_{s}\right) \mathrm{d} \mathbf{x} .
$$

Using a periodizing transformation is equivalent to using a transformed point set, $\mathbf{y}^{(j)} \mapsto\left(\phi\left(y_{1}^{(j)}\right), \ldots, \phi\left(y_{s}^{(j)}\right)\right)$, with weights $w_{j}=\prod_{k=1}^{s} \phi^{\prime}\left(y_{k}^{(j)}\right)$ in (1).

There are several practical problems with periodization. Many periodizing transformations exist. They are mainly used in one dimension and selecting 
the right transform for a given function is not trivial. It seems that the factor $(\log (n))^{\alpha s}$ in the theoretical convergence, as mentioned in Section 2.2, is often very well visible and this gets worse for higher $s$. Consequently more initial points are needed to achieve the $O\left(n^{-\alpha}\right)$.

The periodizing transformations lead to machine dependent cubature rules. When $n$ gets larger, calculations have to be done in higher precision. Indeed, IEEE double precision is not enough since different points map to the same floating point representation even for relatively small $n$; the floating point cube $[0,1]^{s}$ is not symmetric. Furthermore, when $s$ gets higher the weights at the boundaries get very small. Insiders know these problems already a long time. In the recent paper [HR06] this is nicely analyzed.

It follows that periodization is only applicable in low dimensions and with few points. But even then the transformation can give a transformed integrand which is much harder to integrate than before, see [Hic02] for a theoretical discussion and an alternative, and [HR06] for an example. Summarized, periodization is a powerful tool in the hands of an expert but in the hands of the unwary it is a dangerous tool!

\subsection{Lattice Sequences}

In practice one wants to have an error estimate for the approximation. The traditional approach is to use multiple randomly shifted copies of one lattice rule and then using the standard error of the multiple results as a stochastic error estimate [CP76]. However, recent interest is in lattice sequences. These are, not surprisingly, sequences of lattice rules, of which the points are embedded. That is

$$
\Lambda_{0} \subset \Lambda_{1} \subset \cdots \subset \Lambda_{\ell} \subset \cdots .
$$

In this way it becomes possible to obtain an error estimate, e.g., by using the difference of two successive approximations.

Different schemes for this embedding exist. Joe and Sloan [JS92, SJ94] introduced so-called copy rules for this purpose. This idea has been extended in low dimensions to so-called augmented lattice sequences, see, e.g. [HR99, RH02]. A different approach is by using a number of points which is a power of a given integer base. This was done by [HHLL01] and the theoretical existence of good extensible lattice rules was given in [HN03]. Also in [CKN06] and [DPW] such good lattice sequences were successfully constructed.

For such lattice sequences one uses the property that a lattice rule with $b^{m}$ points consists of $b$ smaller lattice rules with $b^{m-1}$ points, which in turn all consist of $b$ smaller lattice rules with $b^{m-2}$ points, and so on. By ordering the points of the biggest lattice rule in a specific way, while keeping the embedding (6) it is even possible to stop anywhere and still have a reasonable good uniform distribution. This fixes one of the historical problems with lattice rules: one can keep on adding points until the error estimate is sufficiently small. An example in two dimensions is given in Figure 1. 


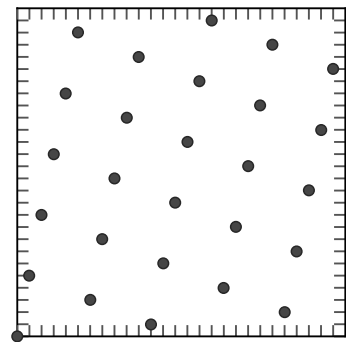

$n=27=3^{3}$

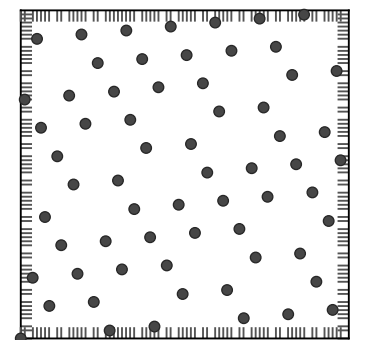

$n=64=2 \times 3^{3}+3^{2}+1$

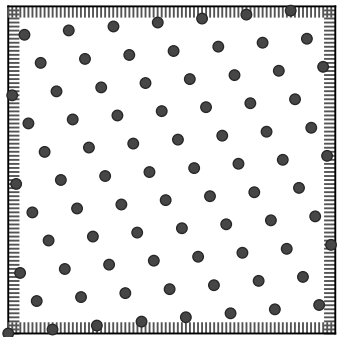

$n=81=3^{4}$

Fig. 1. A lattice sequence in base 3. The first image shows a full lattice using 27 points. The next image shows an extension of this lattice to 64 points, since this is not a power of 3 , the resulting point set is not a full lattice. If we keep on adding points we arrive at the last figure with 81 points, again a full lattice. For a good lattice sequence the intermediate points are well distributed.

\section{Lattice Rules in Action}

In most recent papers on lattice rules, the emphasis is in high dimensions. Let us point out that they are useful and indeed used also in low dimensions, starting from two.

Several general purpose, black-box integration routines for 2-dimensional integration are based on lattice rules. DITAMO [RdD81] is based on the product rectangle rule in combination with the IMT periodizing transformation. d2lri and r2d2lri [HR99, RH02] use augmented lattice rules combined with a periodizing transformation. All these routines are based on sequences of embedded rules and error estimators derived from these. A nice application of 2-dimensional lattice rules is described in [Rev95]. Lattice rules also found applications in the area of computer graphics, see, e.g., [Kel04, DKD08, DK08].

An example of lattice rules in action on a 5-dimensional example is presented in [CN06]. There the result of a lattice rule of high trigonometric degree, constructed along the lines described in Section 3.1, is compared to the result of a lattice rule minimizing some worst-case error, constructed along the lines described in Section 3.2 (and constructed to be a good lattice sequence as described in Section 4). Good results were obtained without the use of periodization and both rules were used as a sequence. Lattice rules and sequences also find applications in much higher dimensions, see, e.g., [CKN06, KDSWW] for examples in 100 and more dimensions.

During the conference several speakers presented results on lattice rules in low and high dimensions. Some of these are included in this volume, e.g., [DKD08, DK08, SJ08]. 


\section{Final Remarks}

The situation of construction methods for lattice rules can be summarized as follows. Searches for lattice rules using the "classical" criteria are doomed to fail for increasing dimensions, not only because the search space is too big but also because the cost for evaluating these criteria is too high. The component-by-component algorithm, relying on the worst-case error for a reproducing kernel Hilbert space beats this curse of dimensionality. It allows the construction of lattice rules very quickly even if $n$ and $s$ are large.

But work remains to be done. For the component-by-component construction, tuning of the function space using the weights must be done so that a given problem belongs to (or is close to) the underlying reproducing kernel Hilbert space. More experience with reliable, cheap and deterministic error estimators for sequences, especially in high dimensions, would be interesting. Currently the usage of a low number of randomizations seems to be the preferred method. We are not aware of any extensive tests for error estimation in high dimensions.

Note that lattice rules are useful for low and high dimensions, and are not only for integrating periodic functions. Furthermore different quality criteria can be useful. Finally the difference between lattice rules and "classical" low discrepancy sequences evaporates. Lattice rules with large $n$ can be constructed easily and can be used as low discrepancy sequences.

We would like to express our hope that some readers want to apply lattice rules in practical problems. We hope that their experiences are positive and that their reports find their way in the growing literature on lattice rules.

\section{Tourist Information}

It is beyond any doubt that the biggest monument in the world devoted to a lattice is the Atomium ${ }^{4}$ in Brussels, Belgium. This monument was designed for the Brussels World's Fair that took place in 1958 (Expo '58). The Atomium consist of 9 balls symbolizing a unit cell of the body centered cubic lattice crystal structure of iron magnified $165 \times 10^{9}$ times.

\section{References}

[Aro50] N. Aronszajn. Theory of reproducing kernels. Trans. Amer. Math. Soc., 68:337-404, 1950.

[BC93] M. Beckers and R. Cools. A relation between cubature formulae of trigonometric degree and lattice rules. In H. Brass and G. Hämmerlin, editors, Numerical Integration IV, pages 13-24, Basel, 1993. Birkhäuser Verlag.

\footnotetext{
${ }^{4}$ See www.atomium.be.
} 
[BP85] M. Bourdeau and A. Pitre. Tables of good lattices in four and five dimensions. Numer. Math., 47:39-43, 1985.

[Coo97] R. Cools. Constructing cubature formulae: the science behind the art, volume 6 of Acta Numerica, pages 1-54. Cambridge University Press, 1997.

[Coo06] R. Cools. More about cubature formulas and densest lattice packings. East Journal on Approximations, 12(1):37-42, 2006.

[CG03] R. Cools and H. Govaert. Five- and six-dimensional lattice rules generated by structured matrices. J. Complexity, 19(6):715-729, 2003.

[CKN06] R. Cools, F. Y. Kuo and D. Nuyens. Constructing embedded lattice rules for multivariate integration. SIAM J. Sci. Comput., 28(6):2162$2188,2006$.

[CL01] R. Cools and J. Lyness. Three- and four-dimensional $K$-optimal lattice rules of moderate trigonometric degree. Math. Comp., 70(236):15491567, 2001.

[CMS01] R. Cools, I. Mysovskikh and H. Schmid. Cubature Formulae and Orthogonal Polynomials. J. Comput. Appl. Math., 127:121-152, 2001.

[CN06] R. Cools and D. Nuyens. The role of structured matrices for the construction of integration lattices. JNAIAM J. Numer. Anal. Ind. Appl. Math., 1(3):257-272, 2006.

[CP76] R. Cranley and T. Patterson. Randomization of number theoretic methods for multiple integration. SIAM J. Numer. Anal., 13:904-914, 1976.

[CS96] R. Cools and I. H. Sloan. Minimal cubature formulae of trigonometric degree. Math. Comp., 65(216):1583-1600, 1996.

[DKD08] H. Dammertz, A. Keller and S. Dammertz. Simulation on rank-1 lattices. In this volume, pages 205-212.

[DK08] S. Dammertz and A. Keller. Image synthesis by rank-1 lattices. In this volume, pages 217-236.

[DK04a] J. Dick and F. Y. Kuo. Constructing good lattice rules with millions of points. In Niederreiter [Nie04], pages 181-197.

[DK04b] J. Dick and F. Y. Kuo. Reducing the construction cost of the component-by-component construction of good lattice rules. Math. Comp., 73(248):1967-1988, 2004.

[DPW] J. Dick, F. Pillichshammer and B. Waterhouse. The construction of good extensible rank-1 lattices. Math. Comp. To appear.

[Fro77] K. Frolov. On the connection between quadrature formulas and sublattices of the lattice of integral vectors. Dokl. Akad. Nauk SSSR, 232:40-43, 1977. (Russian) Soviet Math. Dokl. 18: 37-41, 1977 (English).

[GL87] P. Gruber and C. Lekkerkerker. Geometry of numbers. North Holland, 1987.

[Hic98a] F. J. Hickernell. Lattice rules: How well do they measure up? In P. Hellekalek and G. Larcher, editors, Random and Quasi-Random Point Sets, volume 138 of Lecture Notes in Statistics, pages 109-166. Springer-Verlag, 1998.

[Hic98b] F. Hickernell. A generalized discrepancy and quadrature error bound. Math. Comp., 67(221):299-322, 1998. 
[Hic02] F. J. Hickernell. Obtaining $O\left(n^{-2+\epsilon}\right)$ convergence for lattice quadrature rules. In K. T. Fang, F. J. Hickernell and H. Niederreiter, editors, Monte Carlo and Quasi-Monte Carlo Methods 2000, pages 274-289. SpringerVerlag, 2002.

[HHLL01] F. J. Hickernell, H. S. Hong, P. L'Écuyer and C. Lemieux. Extensible lattice sequences for quasi-Monte Carlo quadrature. SIAM J. Sci. Comput., 22:1117-1138, 2001.

[HN03] F. J. Hickernell and H. Niederreiter. The existence of good extensible rank-1 lattices. J. Complexity, 19(3):286-300, 2003.

[HR99] M. Hill and I. Robinson. d2lri: A nonadaptive algorithm for twodimensional cubature. J. Comput. Appl. Math., 112(1-2):121-145, 1999.

[HR06] M. Hill and I. Robinson. Quadrature using 64-bit IEEE arithmetic for integrands over $[0,1]$ with a singularity at 1 . Theoret. Comput. Sci., 351(1):82-100, 2006.

[Joe04] S. Joe. Component by component construction of rank-1 lattice rules having $O\left(n^{-1}(\ln (n))^{d}\right)$ star discrepancy. In Niederreiter [Nie04], pages 293-298.

[JS92] S. Joe and I. H. Sloan. Embedded lattice rules for multidimensional integration. SIAM J. Numer. Anal., 29:1119-1154, 1992.

[JS07] S. Joe and V. Sinescu. Good lattice rules based on the general weighted star discrepancy. Math. Comp., 76(258):989-1004, 2007.

[Kel04] A. Keller. Stratification by rank-1 lattices. In Niederreiter [Nie04], pages 299-313.

[Kor59] N. Korobov. On approximate calculation of multiple integrals. Dokl. Akad. Nauk SSSR, 124:1207-1210, 1959. (Russian).

[Kor60] N. Korobov. Properties and calculation of optimal coefficients. Dokl. Akad. Nauk SSSR, 132:1009-1012, 1960. (Russian) Soviet Math. Dokl. 1: 696-700, 1960 (English).

[Kuo03] F. Y. Kuo. Component-by-component constructions achieve the optimal rate of convergence for multivariate integration in weighted Korobov and Sobolev spaces. J. Complexity, 19:301-320, 2003.

[KDSWW] F. Y. Kuo, W. T. M. Dunsmuir, I. H. Sloan, M. P. Wand and R. S. Womersley. Quasi-Monte Carlo for highly structured generalised response models. Methodology and Computing in Applied Probability. To appear.

[LL02] P. L'Écuyer and C. Lemieux. Recent advances in randomized quasiMonte Carlo methods. In M. Dror, P. L'Ecuyer and F. Szidarovszki, editors, Modeling Uncertainty: An Examination of Stochastic Theory, Methods, and Applications, pages 419-474. Kluwer Academic Publishers, 2002 .

[Lyn89] J. Lyness. An introduction to lattice rules and their generator matrices. IMA J. Numer. Anal., 9:405-419, 1989.

[Lyn03] J. Lyness. Notes on lattice rules. J. Complexity, 19(3):321-331, 2003.

[LS04] J. Lyness and T. Sørevik. Four-dimensional lattice rules generated by skew-circulant matrices. Math. Comp., 73(245):279-295, 2004.

[LS06] J. Lyness and T. Sørevik. Five-dimensional $k$-optimal lattice rules. Math. Comp., 75(255): 1467-1480, 2006. 
[Mai72] D. Maisonneuve. Recherche et utilisation des "bons treillis". Programmation et résultats numerériques. In S. Zaremba, editor, $A p$ plications of Number Theory to Numerical Analysis, pages 121-201. Academic Press, 1972.

[Min67] H. Minkowski. Gesammelte Abhandlungen. Chelsea Publishing Company, New York, Reprinted (originally published, in 2 volumes, Leipzig, 1911) edition, 1967.

[Mys81] I. Mysovskikh. Interpolatory Cubature Formulas. Izdat. 'Nauka', Moscow-Leningrad, 1981. (Russian).

[Mys85] I. Mysovskikh. Quadrature formulae of the highest trigonometric degree of accuracy. Zh. vychisl. Mat. mat. Fiz., 25:1246-1252, 1985. (Russian) U.S.S.R. Comput. Maths. Math. Phys. 25:180-184, 1985 (English).

[Mys87] I. Mysovskikh. On cubature formulas that are exact for trigonometric polynomials. Dokl. Akad. Nauk SSSR, 296:28-31, 1987. (Russian) Soviet Math. Dokl. 36:229-232, 1988 (English).

[Mys90] I. Mysovskikh. On the construction of cubature formulas that are exact for trigonometric polynomials. In A. Wakulicz, editor, Numerical Analysis and Mathematical Modelling, volume 24 of Banach Center Publications, pages 29-38. PWN - Polish Scientific Publishers, Warsaw, 1990. (Russian).

[NC06a] D. Nuyens and R. Cools. Fast algorithms for component-by-component construction of rank-1 lattice rules in shift-invariant reproducing kernel Hilbert spaces. Math. Comp., 75(2):903-920, 2006.

[NC06b] D. Nuyens and R. Cools. Fast component-by-component construction of rank-1 lattice rules with a non-prime number of points. J. Complexity, 22(1):4-28, 2006.

[Nie78] H. Niederreiter. Quasi-Monte Carlo methods and pseudo-random numbers. Bull. Amer. Math. Soc., 84(6):957-1041, 1978.

[Nie92] H. Niederreiter. Random Number Generation and Quasi-Monte Carlo Methods, volume 63 of CBMS-NSF regional conference series in applied mathematics. SIAM, Philadelphia, 1992.

[Nie04] H. Niederreiter, editor. Monte-Carlo and Quasi-Monte Carlo Methods 2002. Springer-Verlag, 2004.

[Nos85] M. Noskov. Cubature formulae for the approximate integration of periodic functions. Metody Vychisl., 14:15-23, 1985. (Russian).

[Nos88] M. Noskov. Formulas for the approximate integration of periodic functions. Metody Vychisl., 15:19-22, 1988. (Russian).

[Osi04] N. N. Osipov. Cubature formulas for periodic functions. Ph.D. thesis, Krasnoyarsk State Technical University, 2004. (Russian).

[OP04] N. N. Osipov and A. V. Petrov. Construction of sequences of lattice rules which are exact for trigonometric polynomials in four variables. Vychisl. Tekhnol., 9, Spec. Iss. 1:102-110, 2004. (Russian).

[Rad48] J. Radon. Zur mechanischen Kubatur. Monatsh. Math., 52:286-300, 1948.

[RdD81] I. Robinson and E. deDoncker. Algorithm 45: Automatic computation of improper integrals over a bounded or unbounded planar region. Computing, 27:253-284, 1981.

[Rev95] M. Revers. Numerical integration of the Radon transform on classes $E_{s}^{\alpha}$ in multiple finite dimensions. Computing, 54(2):147-165, 1995. 
[Rez90] A. Reztsov. On cubature formulas of Gaussian type with an asymptotic minimal number of nodes. Mathematicheskie Zametki, 48:151-152, 1990.

[RH02] I. Robinson and M. Hill. Algorithm 816: r2d2lri: an algorithm for automatic two-dimensional cubature. ACM Trans. Math. Software, 28(1):75-100, 2002.

[Sem96] A. Semenova. Computing experiments for construction of cubature formulae of high trigonometric accuracy. In M. Ramazanov, editor, Cubature Formulas and their Applications (Russian), pages 105-115, Ufa, 1996.

[Sem97] A. Semenova. An algorithm for the construction of cubature formulas of high trigonometric accuracy. In C. Shoynjurov, editor, Cubature Formulas and their Applications (Russian), pages 93-105, Ulan-Ude, 1997.

[SJ08] V. Sinescu and S. Joe. Good lattice rules with a composite number of points based on the product weighted star discrepancy. In this volume, pages $645-658$.

[SJ94] I. H. Sloan and S. Joe. Lattice Methods for Multiple Integration. Oxford University Press, 1994.

[SK87] I. H. Sloan and P. Kachoyan. Lattice mathods for multiple integration: theory, error analysis and examples. SIAM J. Numer. Anal., 24:116-128, 1987.

[SKJ02a] I. H. Sloan, F. Y. Kuo and S. Joe. Constructing randomly shifted lattice rules in weighted Sobolev spaces. SIAM J. Numer. Anal., 40(5):1650$1665,2002$.

[SKJ02b] I. H. Sloan, F. Y. Kuo and S. Joe. On the step-by-step construction of quasi-Monte Carlo integration rules that achieve strong tractability error bounds in weighted Sobolev spaces. Math. Comp., 71(240):16091640, 2002.

[SR02] I. H. Sloan and A. V. Reztsov. Component-by-component construction of good lattice rules. Math. Comp., 71(237):263-273, 2002.

[Str60] A. Stroud. Quadrature methods for functions of more than one variable. New York Acad. Sci., 86:776-791, 1960.

[Tem91] N. Temirgaliev. Application of divisor theory to the numerical integration of periodic functions of several variables. Math. USSR Sbornik, 69(2):527-542, 1991. 